\title{
ACTUACIONES FORESTALES DE LA COMUNIDAD ECONÓMICA EUROPEA: EL EJEMPLO DEL DEPARTAMENTO FRANCÉS DE BOUCHES-DU-RHONE
}

\author{
Cristina Montiel Molina
}

\section{RESUMEN}

La protección del bosque mediterráneo, ante el proceso de progresiva degradación ecológica, y frente a la constante amenaza de los incendios forestales, constituye uno de los objetivos fundamentales de la ayuda que presta la Comunidad Económica Europea en favor del medioambiente. El departamento francés de Bouches-du-Rhône viene siendo beneficiario de este tipo de acciones comunitarias a partir de 1980, en el marco del I Plan Quinquenal F.E.O.G.A. y de los Programas Integrados Mediterráneos, aplicados por primera vez en el departamento en 1987.

En este sentido, la ayuda económica prestada por las Comunidades Europeas ha permitido el desarrollo de una eficaz política de protección y de mejora forestal, basada en la repoblación de terrenos desprovistos de vegetación, en la regeneración de formaciones edáficas y vegetales degradadas, en la ordenación forestal y en la defensa de los montes contra los incendios.

\section{RESUMÉ}

La défense de la forêt méditerranéenne contre la dégradation écologique et contre la ménace permanente des feux de forêt c'est l'un des objectifs principaux des aides de la Communauté Economique Européenne en matière d'environement. Le département français des Bouches-du-Rhône bénéficie de ces actuations communautaires depuis 1980, dans le cadre du I Plan Quinquennal F.E.O.G.A. et des Programmes Intégrés Méditerranéens, appliqués par la première fois dans le département en 1987.

Les subventions des Communautés Européennes ont permis le développement d'une politique de protection et d'amélioration de la forêt, appuyée sur le reboisement des formations édaphiques et végétales dégradées, sur l'aménagement forestier et sur la protection des forêts contre l'incendie.

\section{Orientaciones de la política forestal en la región mediterránea}

A pesar de que las directrices de la política francesa en materia forestal tienden a incrementar y a mejorar la producción de los montes, el objetivo prioritario de la gestión 


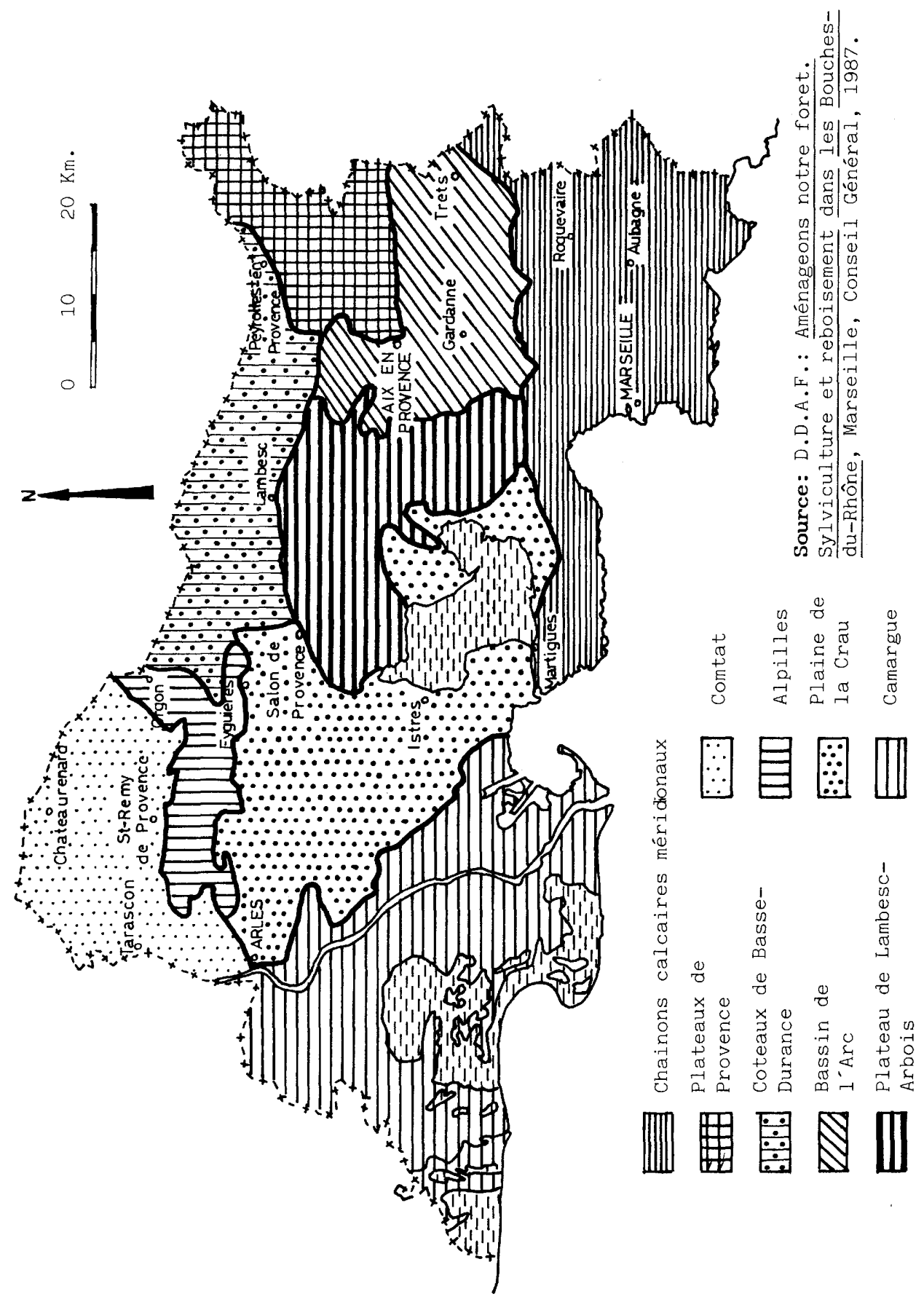

FIGURA 1

Regiones forestales del departamento de Bouches-du-Rhône. 
forestal en el departamento mediterráneo de Bouches-du-Rhône es la recuperación y el mantenimiento del equilibrio ecológico en los espacios naturales. La explicación a esta orientación específica de la política forestal reside en los condicionamientos negativos que, desde el punto de vista físico, ofrece la región mediterránea al desarrollo de formaciones vegetales rentables para el aprovechamiento silvícola.

La protección del bosque mediterráneo, ante el proceso de progresiva degradación ecológica, y frente a la constante amenaza de los incendios forestales, constituye uno de los objetivos fundamentales de las ayudas financieras que presta la Comunidad Económica Europea en favor del medioambiente. Sin duda, uno de los mejores ejemplos con que podríamos ilustrar este tipo de acciones es la contribución de los fondos comunitarios, a partir de 1980, a la financiación de los trabajos forestales en el departamento de Bouchesdu-Rhône. El punto de partida de la acción comunitaria en el sector forestal de dicho departamento fue la publicación del Reglamento C.E.E. n. $\stackrel{\circ}{269 / 79}$ del Consejo del 6 de febrero de 1979 en el Boletín Oficial de las Comunidades Europeas del 14 de febrero del mismo año.

La ayuda económica prestada por las Comunidades Europeas ha permitido a los organismos encargados de la gestión de los montes el desarrollo de una adecuada política de protección medioambiental, de regeneración de formaciones edáficas y vegetales degradadas, y de ordenación del territorio en el sector forestal. Efectivamente, las operaciones realizadas en Bouches-du-Rhône durante el período 1980-1985, bajo los auspicios del I Plan Quinquenal del Fondo Europeo de Orientación y Garantía Agrícola (F.E.O.G.A.), tuvieron como objetivo la ejecución de repoblaciones forestales, con el fin de garantizar la conservación de suelos, la regularización del régimen hidrológico y el desarrollo de los aprovechamientos forestales. A tal efecto, la Comisión de Comunidades Europeas estableció una relación de apartados especificando el tipo de trabajos cuya ejecución podría beneficiarse de la subvención de los fondos comunitarios. Dicha relación incluía los trabajos de repoblación forestal en terrenos desprovistos de vegetación, las tareas de mejora de bosques degradados, la creación de pistas y caminos de acceso forestales, los trabajos auxiliares a las operaciones de repoblación y mejora, y los trabajos de defensa contra incendios forestales.

\section{Balance del I Plan Quinquenal del F.E.O.G.A.}

En el departamento de Bouches-du-Rhône la subvención de proyectos de repoblación forestal ha supuesto el $26 \%$ de las inversiones de los fondos comunitarios, mientras que los trabajos de mejoras representan un porcentaje del $65,3 \%$. Estos últimos han consistido fundamentalmente en la realización de podas y clareos en formaciones resinosas, en la transformación del monte bajo en formaciones arbóreas frondosas o resinosas mediante la introducción de nuevas especies vegetales, y en la zonificación y amojonamiento de áreas boscosas y zonas dedicadas a pasto para el ganado. Con respecto a las repoblaciones forestales, las especies más empleadas han sido el cedro del Atlas y el pino de Alepo.

Los trabajos auxiliares, en otro orden de cosas, han supuesto el 1,61\% de las inversiones, y han consistido en la creación de aterrazamientos de ladera y en la realización de toda una serie de obras tendentes a la conservación y a la estabilización de las formaciones edáficas, en tanto en cuanto estas labores garantizan el éxito de los trabajos de repoblación y mejora forestal. Las terrazas han sido construidas fundamentalmente en las zonas más afectadas por los procesos erosivos, tales como las arcillas rojas del macizo del Cengle.

Finalmente, a la protección de los montes contra los incendios se ha dedicado el 5,70\% de las inversiones totales de los fondos comunitarios, lo que ha permitido la realización de 


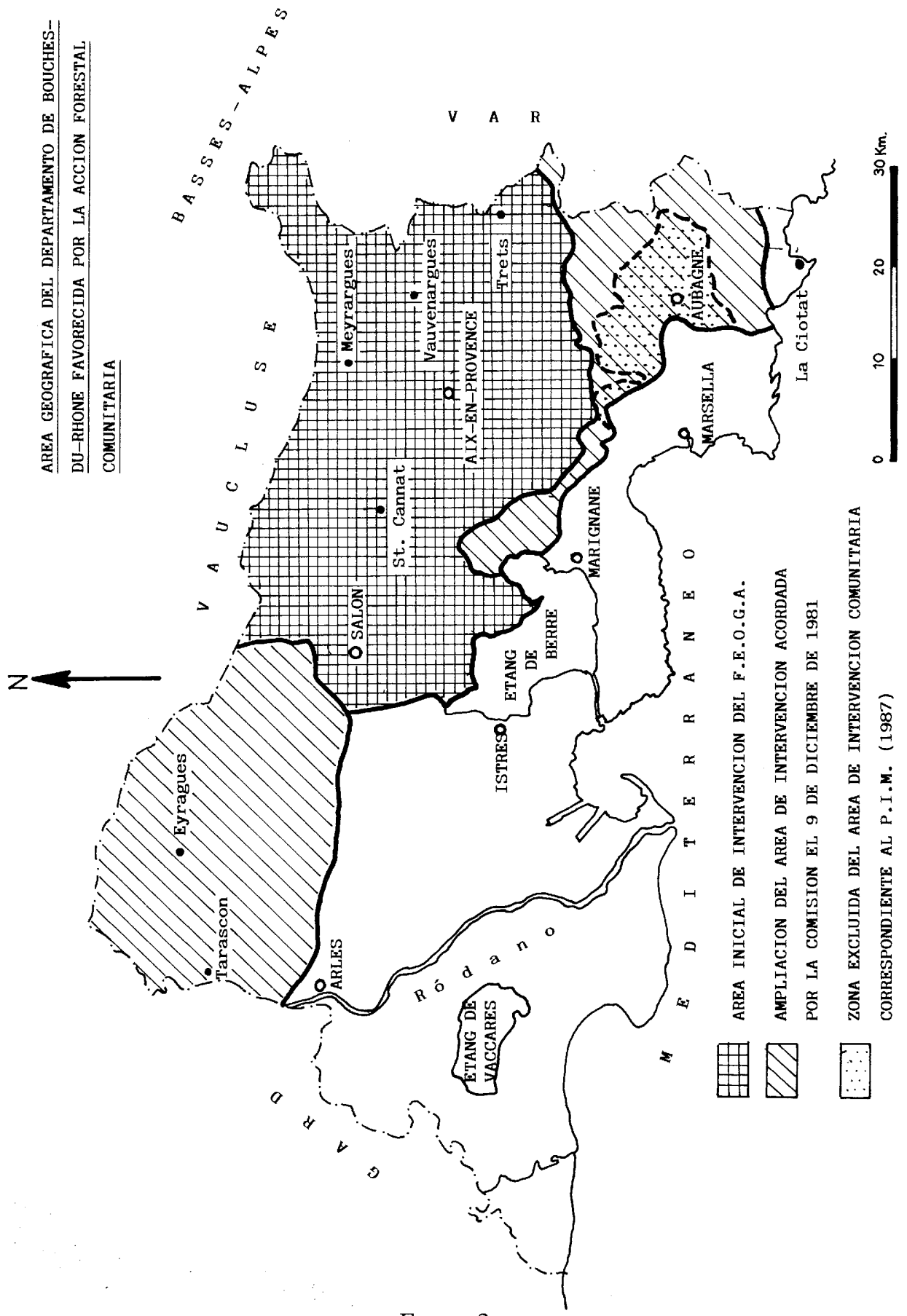

FIgURA 2

Área geográfica del departamento favorecida por la acción forestal comunitaria. 
trabajos en apoyo de la lucha preventiva en 3.338 ha. de superficie forestal de Bouches-duRhône. Estos trabajos han consistido esencialmente en la creación de fajas auxiliares desbrozadas, tendentes a limitar el riesgo de propagación de incendios, y en la instalación de cisternas de agua.

Conviene señalar que el departamento de Bouches-du-Rhône no ha sido en su totalidad favorecido por la política de ayuda comunitaria en materia forestal. En efecto, la franja litoral y el norte del Comtat no fueron inicialmente incluidos en el área de intervención del programa F.E.O.G.A. Y ello se debe al tipo de criterio establecido para definir el área favorecida por la acción forestal comunitaria. Éste ha consistido en la influencia que ejerce el estado de las masas forestales sobre la agricultura de los sectores bajos, de modo que las áreas inicialmente favorecidas han sido las partes altas de las cuencas vertientes que avenan valles de predominio agrícola (cuenca vertiente del Durance, del Arc y del Touloubre). Sin embargo, la Comisión de la C.E.E. decidió el 9 de febrero de 1981 ampliar la extensión del área de intervención comunitaria, abarcando el sur de la cuenca del Huveaune y del Jarret, las planicies del este de Marsella, la parte meridional de los macizos situados al este del departamento (Regagnas, Sainte-Baume, Etoile, Garlaban, Fontblanche et Saint-Cyr), la cuenca de Cuges-les-Pins, Roquefort-la-Bedoule, Casis y la Ciotat, el Comtat y el norte de la Crau. Además, el Ministerio de Agricultura advirtió también en 1981 que no serían aceptados los proyectos de repoblación o mejora forestal en superficies de extensión inferior a las diez hectáreas, y que el propietario debía necesariamente comprometerse por escrito al mantenimiento del uso forestal de los terrenos durante el período mínimo de treinta años.

Los titulares de terrenos forestales que pueden en principio participar de las ayudas financieras comunitarias son el Estado, los municipios o «communes», el departamento, los entes públicos y las asociaciones de propietarios.

Con respecto a los montes estatales, es interesante destacar que la mayor parte de los fondos asignados se ha dedicado a la mejora de los bosques degradados y a la defensa contra los incendios forestales. Merece la pena igualmente subrayar que el volumen más importante de trabajos de repoblación forestal llevados a cabo en terrenos desprovistos de vegetación, y de construcción de caminos forestales, se ha realizado en bosques pertenecientes a las «communes». A estas últimas ha correspondido el $40,8 \%$ de las inversiones totales relativas al I Plan Quinquenal, mientras que la cantidad asignada a los montes departamentales ha representado el $6,45 \%$ y el organismo departamental encargado de la lucha contra incendios forestales ha recibido el $8,37 \%$ de las inversiones.

\section{El enfoque integrador de los P.I.M.}

En 1986, los montes del departamento de Bouches-du-Rhône no contaron con ningún tipo de ayuda comunitaria, debido a que el I Plan Quinquenal del F.E.O.G.A. había concluido sin que hubiera sido previsto con antelación la elaboración de un nuevo programa de subvenciones. Éste fue finalmente concebido bajo la denominación de «Programa Integrado Mediterráneo (P.I.M.)», y aplicado por primera vez en Bouches-du-Rhône en 1987. El objetivo fundamental, que marca las directrices de la elaboración y aplicación del P.I.M., es el propósito de acometer de un modo global los programas de desarrollo que afectan a la agricultura y al resto de los sectores de actividad económica de una región. De acuerdo con este criterio, la Comisión de las Comunidades Europeas adoptó el 21 de diciembre de 1983 (Boletín Oficial de las Comunidades Económicas Europeas del 15 de febrero de 1984) la decisión de instaurar una acción piloto en la región de Córcega, con el 
fin de preparar la puesta en práctica de los Programas Mediterráneos Integrados en el resto de la cuenca. Se trataba de una acción basada en el logro del desarrollo agro-silvo-pastoral de una superficie de 10.000 hectáreas que, en materia forestal, contemplaba la defensa contra incendios forestales, la mejora de las formaciones boscosas existentes y la revalorización de la producción forestal local.

El P.I.M. que ha sustituido al Plan Quinquenal F.E.O.G.A. en el departamento de Bouches-du-Rhône es, en definitiva, un conjunto de medidas tendentes a incrementar la superficie de bosques y la producción forestal. De acuerdo con el pliego de prescripciones técnicas de este programa, el objetivo fundamental es alcanzar la revalorización del monte, partiendo de la base de que éste es el medio más apropiado para garantizar la protección del medioambiente. Por otra parte, el reglamento considera que las decisiones y proyectos han de ser ejecutados a escala de macizos o conjuntos montañosos, y no en función de criterios administrativos o de titularidad, con el fin de lograr una mayor eficacia y rentabilidad. El P.I.M. de Bouches-du-Rhône apunta asimismo la conveniencia de prever el mantenimiento de los trabajos de ordenación del territorio y, en definitiva, de conceder la prioridad a la subvención de proyectos relativos a montes que ofrezcan garantías de una gestión apropiada, tales como los montes de particulares que cuentan con un Plan de Gestión, los montes de las «communes» sometidos al régimen forestal y administrados por la Oficina Nacional de Montes (Office National de Forêts, O.N.F.) - homólogo del I.C.O.N.A. en España-, y los montes incluidos en Planes Comarcales de Desarrollo y de Ordenación Forestal, entre otros. Por otra parte, el área geográfica de Bouches-du-Rhône afectada por el P.I.M. difiere de la anteriormente contemplada por el F.E.O.G.A., ya que han sido excluidas Marsella y su área de influencia.

El programa P.I.M. correspondiente al año 1988 ha supuesto en Bouches-du-Rhône una inversión total de 24.182 francos, distribuidos del siguiente modo:

\begin{tabular}{|c|c|c|}
\hline TRABAJOS & SUPERFICIE (ha.) & IMPORTE (francos) \\
\hline 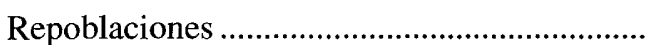 & 351,5 & 5.587 .000 \\
\hline 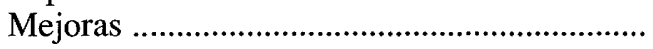 & 535,0 & 6.703 .000 \\
\hline Defensa contra & & \\
\hline , & $11.325,0$ & 6.167 .000 \\
\hline Trabajos auxiliares & $1.158,5$ & 5.725 .000 \\
\hline TITULARES & \multicolumn{2}{|l|}{ IMPORTE (francos) } \\
\hline 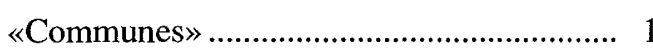 & \multicolumn{2}{|c|}{13.090 .000} \\
\hline 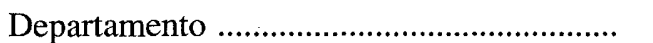 & \multicolumn{2}{|l|}{1.040 .000} \\
\hline Asociaciones de propietarios ............................ & \multicolumn{2}{|l|}{8.562 .000} \\
\hline Estado & \multicolumn{2}{|l|}{600.000} \\
\hline 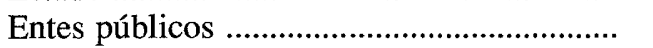 & \multicolumn{2}{|l|}{890.000} \\
\hline
\end{tabular}

\section{Conclusión}

A modo de síntesis, podemos presentar las siguientes tablas, que reflejan la cuantía, evolución y distribución de la ayuda económica comunitaria para la protección de terrenos forestales en el departamento de Bouches-du-Rhône. Las cifras expuestas representan el $50 \%$ del importe total de los trabajos forestales realizados en dicho departamento, lo que 
nos permite deducir la importancia absoluta y relativa de estas cantidades. Efectivamente, la contribución de la C.E.E. a la financiación de determinado tipo de trabajos forestales ha posibilitado a los organismos encargados de la gestión de los montes el desarrollo de una eficaz política de protección y de mejora forestal.

Podemos afirmar, sin lugar a dudas, que en el caso del sector forestal de Bouches-duRhône, la aplicación de los instrumentos económicos-financieros comunitarios posee una importancia de primer orden para la protección del medioambiente. Sin la ayuda económica comunitaria, dicho departamento no hubiera podido emprender toda una serie de acciones y programas que han contribuido a la mejora y ordenación de sus montes y a la disposición de unos medios de defensa más adecuados para la lucha contra la degradación causada por la intervención antrópica. El departamento de Bouches-du-Rhône constituye, pues, en este sentido, un modelo a tener en cuenta por los organismos responsables de la política forestal en España, en un momento en que nuestro país cuenta con la posibilidad de acceder a este tipo de ventajas económico-financieras. 


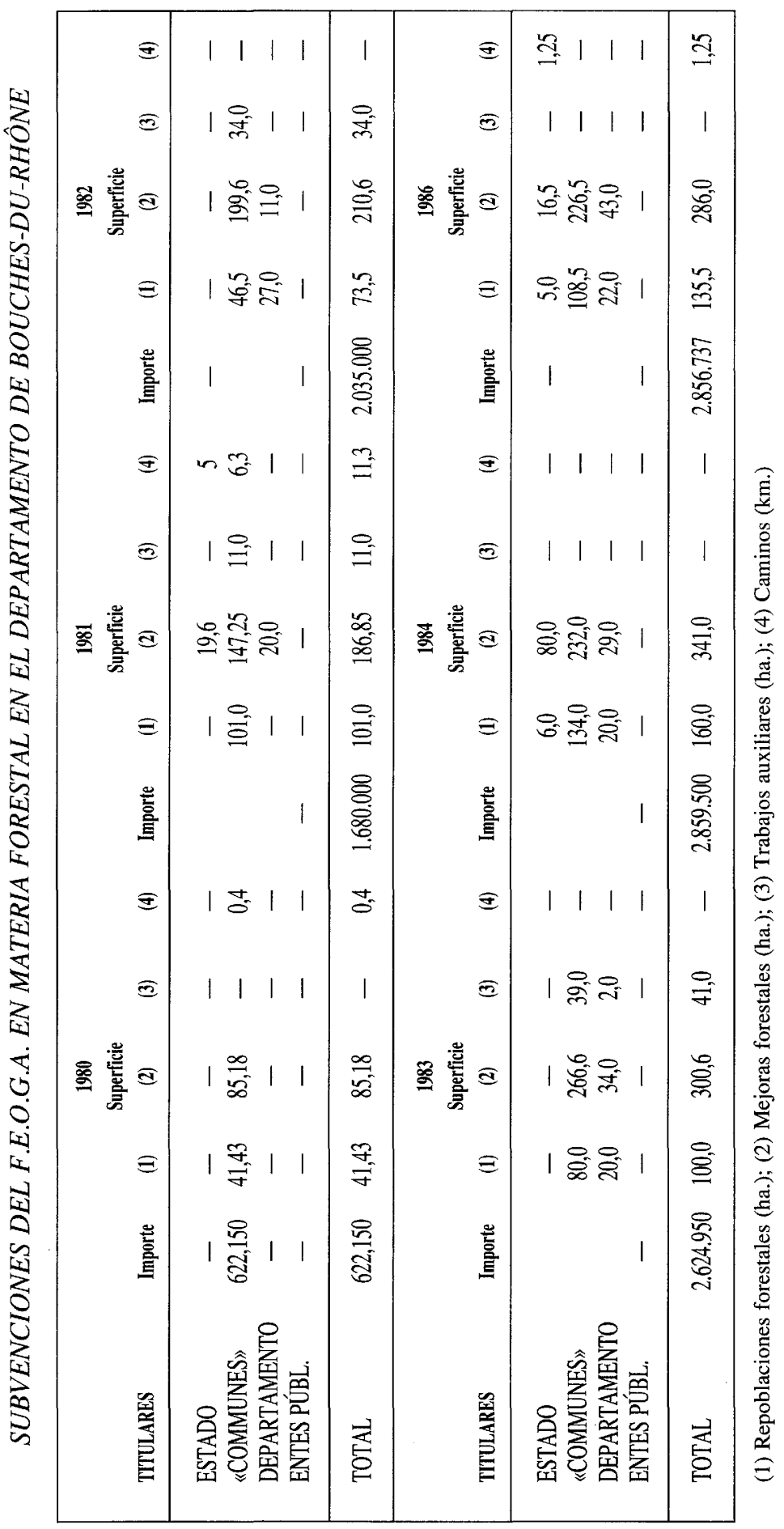




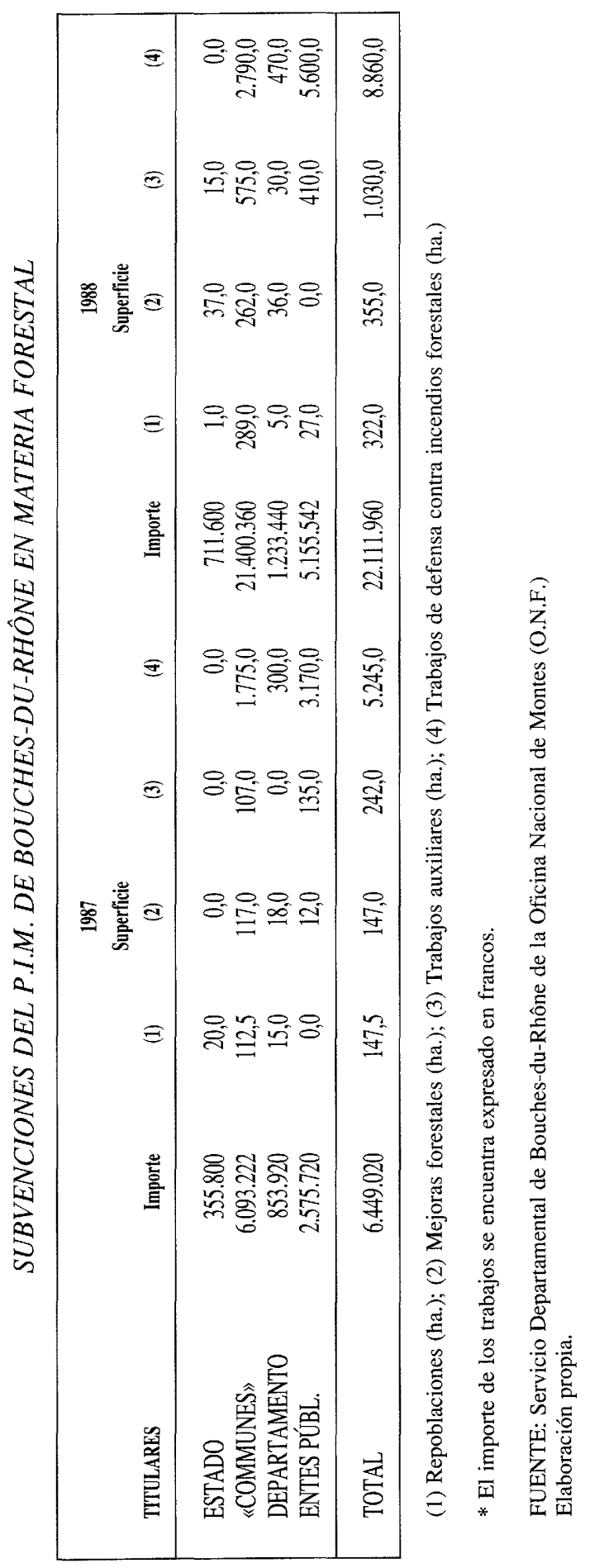

